\title{
El aprendizaje a través de la ciencia ficción: El audiovisual Guardians of the heritage
}

\section{Learning through science fiction: The guardians of the heritage audiovisual}

\section{Francisca Ramón Fernández}

frarafer@urb.upv.es

https://orcid.org/0000-0002-0936-8229

Vicent Giménez Chornet

vigicho@har.upv.es

https://orcid.org/0000-0003-1183-9058

Vicente Cabedo Mallol

vicamal@urb.upv.es

https://orcid.org/0000-0001-5248-5220

Juan Vicente Oltra Gutiérrez

jvoltra@omp.upv.es

https://orcid.org/0000-0002-7765-1085

Universitat Politécnica de Valéncia, Valencia, España

Recibido: $23 / 01 / 21$

Aceptado: 10/05/21

\section{Resumen}

Se presenta la innovación y resultados obtenidos de la utilización de un audiovisual de ciencia ficción "Guardians of the heritage" con la finalidad de favorecer el aprendizaje de la asignatura Gestión del documento electrónico. El objetivo del artículo es analizar si los alumnos han valorado positivamente la visualización de un audiovisual relacionado con un contenido de la asignatura, y si de esta forma los conceptos explicados en clase se pueden asimilar mejor.

Palabras clave: ciencia ficción; aprendizaje; competencias; innovación; audiovisual.

\section{Abstract}

The innovation and results obtained from the use of a science fiction audiovisual "Guardians of the eritage" are presented in order to favor the learning of the subject Electronic document management. The aim of the article is to analyze if the students have positively valued the visualization of an audiovisual related to the content of the subject, and if in this way the concepts explained in class can be better assimilated. 
Keywords: ccience fiction, learning, skills, innovation, audiovisual.

\section{Introducción}

Uno de los retos de los profesores de educación superior sobre administración pública es hacer visualizar a los estudiantes una profesión poco conocida. Entre los contenidos docentes se encuentra el aprendizaje de la gestión de los documentos generados por las administraciones. Aunque en el último curso el alumno tiene la posibilidad de realizar prácticas docentes en alguna administración, en los cursos previos el profesor necesita trasladar a los alumnos la problemática de gestionar los documentos que se han creado, y que son fundamentales como registro de las evidencias de los actos administrativos que posiblemente en un futuro serán necesarios para probar actos, ejercer a transparencia de la administración pública, o simplemente poner en valor las acciones y relevancia de la entidad (Montero Caro, 2020). Uno de los principales retos de las instituciones de enseñanza superior es conseguir un aprendizaje basado en la experiencia del trabajo laboral, dado que es una prioridad cada vez más alta para los empleadores y el gobierno. En este aprendizaje las instituciones de enseñanza superior deben acreditar y ampliar las competencias y habilidades laborales de sus estudiantes (Hollist, 2012). Una opción para que los alumnos experimenten un contexto laboral es la visualización de un vídeo que les permita reflexionar sobre una actividad concreta del trabajo en las empresas o la administración pública.

La utilización de recursos audiovisuales en el aula es uno de los principales propósitos del grupo EICE RETAJUDOCA (Recursos tecnológicos para el aprendizaje jurídico, la documentación y la comunicación audiovisual) de la Universitat Politècnica de València. Ha sido frecuente el uso de audiovisuales en la enseñanza de idiomas (Abreus González, \& Haro Calero, 2019), pero poco frecuente en la docencia de otros contenidos no ligados al lenguaje. Actualmente estamos desarrollando de recursos y en la utilización de audiovisuales relacionados con la ciencia ficción den el marco del PIME "Gamificación y TICs: diseño de actividades audiovisuales basadas en la ciencia ficción para la dinamización docente en un entorno presencial, semipresencial y virtual".

En la presente experiencia que vamos a mostrar nos hemos centrado en un recurso audiovisual de libre acceso, en youtube (https://www.youtube.com/watch?time_continue $=106 \& v=s O h W s N J 8 U u o \&$ feature $=e m b$ logo) dentro del repositorio de la Universidad de Alcalá, titulado Guardians of the Heritage con la finalidad de ayudar en el aprendizaje de los conceptos de la asignatura "Gestión del documento electrónico" que se imparte en el Grado de Gestión y Administración Pública en la Facultad de Administración y Dirección de Empresas, de la Universitat Politècnica de València, por parte del área de Biblioteconomía y Documentación del Departamento de Comunicación Audiovisual, Documentación e Historia del Arte. Previo al documental se impartió una clase sobre el cambio de paradigma en la gestión de los documentos generados por las administraciones públicas (Sebastia Salat, 2009), de unas técnicas de gestión de documentos en papel a otras tecnologías necesarias para 
la gestión de los documentos electrónicos. Se pretende transmitir a los alumnos que la implantación de productos y servicios innovadores en los archivos ha sido posible gracias a la incorporación de las recientes funcionalidades en las Tecnologías de la Información y la Comunicación (Giménez Chornet, 2014).

\section{Objetivo y metodología}

El objetivo del artículo es analizar si los alumnos han valorado positivamente la visualización de un audiovisual relacionado con un contenido de la asignatura, y si de esta forma los conceptos explicados en clase se pueden asimilar mejor.

El audiovisual Guardians of the Heritage que se encuentra disponible en el repositorio de la Universidad de Alcalá, en fecha 22 de febrero de 2019. Como se indica en la presentación del audiovisual, "A lo largo de los siglos, la Universidad de Alcalá ha conservado su patrimonio documental. En el Archivo de la Universidad, protegemos este patrimonio para garantizar el cumplimiento normativo y el conocimiento de la historia de la Universidad. Además, la llegada de Internet y las TIC ha ayudado enormemente a mejorar el tratamiento y la preservación de documentos y el acceso a la información. Somos los guardianes de la herencia" (Figura 1).

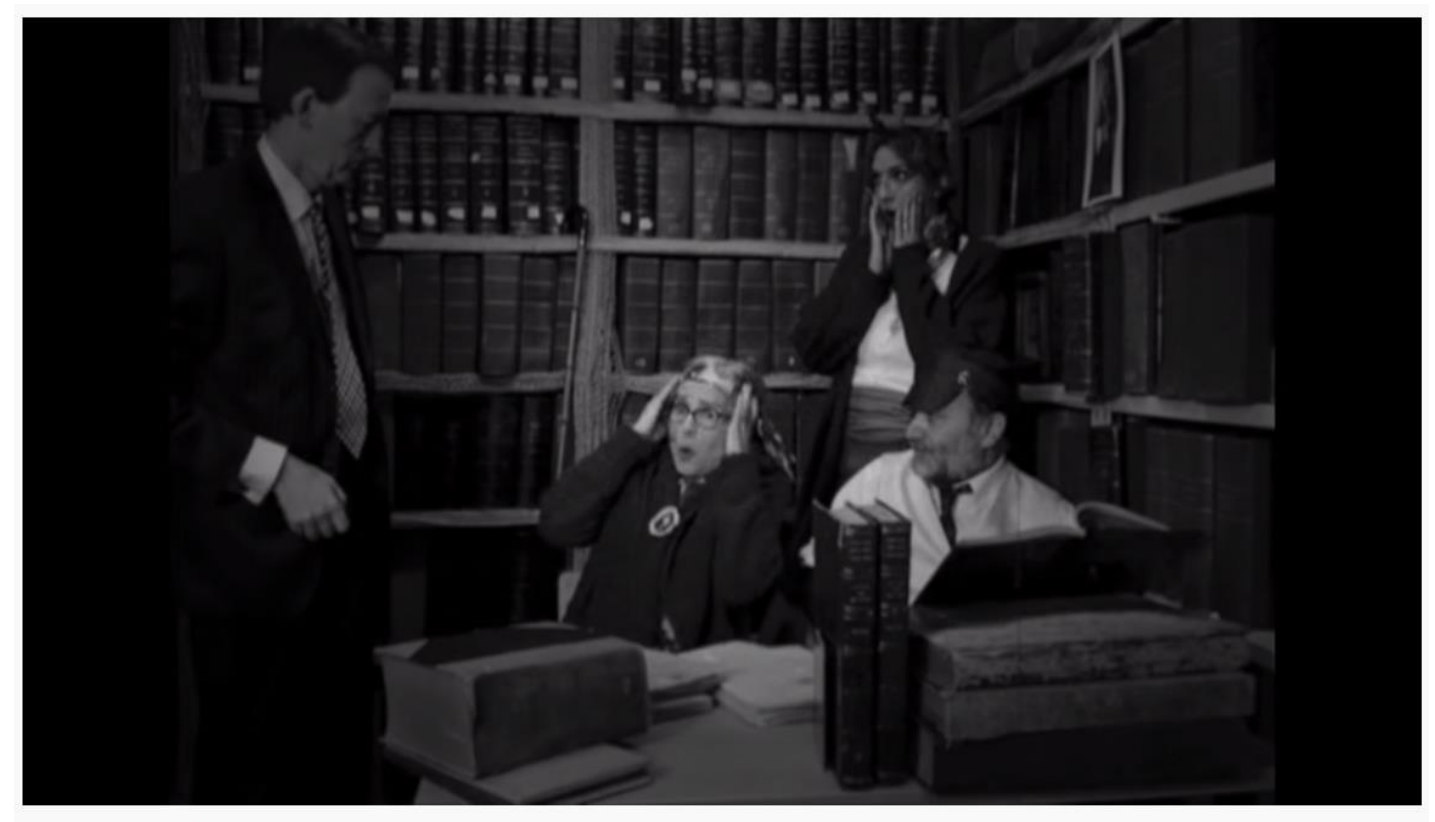

Guardians of the Heritage

Figura. 1. Captura de pantalla de Guardians of the Heritage. Fuente: youtube. <https://www.youtube.com/watch?time_continue $=106 \&$ v $=$ sOhWsNJ8Uuo\&feature $=e m b$ _logo> [Consulta: 25 de enero de 2021].

La razón de elección de dicho audiovisual radica en varios factores ligados al documento electrónico que nos interesan para el desarrollo de la innovación docente: 
a) Muestra la evolución del tratamiento de los documentos antiguos.

b) Finalidad específica de dar a conocer las dificultades de localización de los documentos más antiguos en un sistema tradicional y no informatizado.

c) Exposición mediante un audiovisual de cine mudo en los primeros minutos, las peripecias de los archiveros ante las dificultades de gestión de los documentos no electrónicos.

d) Audiovisual que muestra notas de ciencia ficción una situación que se daba en los archivos hasta hace poco tiempo, antes de la informatización y las bases de datos, para mostrar, después, las nuevas tecnologías de la información y comunicación (TICs) y la importancia de la preservación digital.

e) Hacer referencia a la importancia del patrimonio documental en la legislación aplicable.

Tratamos de utilizar el audiovisual para que el alumnado vea en tono de ciencia ficción la evolución informática en el ámbito de la documentación, así como que pueda comprender de forma más clara la importancia de la informática como herramienta útil y ágil en la conservación y difusión de la información contenida en documentos antiguos.

Para conocer el alcance de la experiencia docente se ha diseñado una encuesta específica para valorar la mejora del aprendizaje de conceptos por parte de los alumnos con los siguientes objetivos:

a) Utilización de un audiovisual relacionado con la documentación, con notas de ciencia ficción, siguiendo la actividad del PIME que actualmente estamos desarrollando.

b) Incrementar el aprendizaje del alumnado en los conceptos y características relacionados con la transformación en la gestión del documento electrónico.

c) Relacionar el audiovisual con otros audiovisuales que traten sobre la gestión del documento electrónico.

d) Observar si el alumnado concede importancia a las TIC como medio de difusión del conocimiento respecto a la gestión del documento electrónico.

El pequeño grupo de alumnos de la asignatura optativa donde se ha realizado la experiencia lleva asociado el punto de control de unas competencias orientadas a la futura actividad profesional, y son las siguientes:

1. Que sean capaces de gestionar la información, delimitando su importancia y aplicación en el entorno profesional.

2. Capacidad de comunicación oral y escrita con un lenguaje comprensivo.

3. Saber gestionar la información y documentación administrativa.

4. Resolución de problemas multidisciplinares de forma coherente y aportar soluciones creativas en la resolución de los problemas.

La competencia transversal que se trabaja y que es punto de control es la de "Diseño y proyecto", con la que se pretende que el alumnado conozca el procedimiento 
administrativo electrónico, así como la legislación de reutilización de la información. También se les formará en el uso del certificado digital y el análisis del mapa de documentos, junto con los elementos de la tramitación electrónico. Se pretende trasladar también una reflexión propia en el análisis de contenidos.

La utilización de series, películas o fragmentos audiovisuales, en definitiva, potencia el aprendizaje del alumnado como se ha puesto de manifiesto en distintas ocasiones a través de distintas experiencias de innovación docente (Cabedo el al., 2016, 2017, 2018 y 2019). El impacto visual que aporta la imagen propicia un incremento interés en el aula, así como constituye una herramienta que favorece la dinamización (Oltra et al., 2016; Ramón et al., 2016a y b; 2018a, b y c; 2019 a y b).

\section{Desarrollo de la innovación y su aplicación al Grado de Gestión Administrativa de la Universitat Politècnica de València}

En la asignatura de "Gestión del documento electrónico" el alumnado conoce los fundamentos de la gestión de los documentos digitales, con un componente práctico a través de los portales web de administraciones públicas, metadatos y procedimientos y expedientes electrónicos.

Para que los alumnos reflexionaran sobre el audiovisual Guardians of the Heritage, iniciamos un debate en torno a las siguientes cuestiones:

1. ¿Cómo buscaban los documentos en la época antigua?

2. En la segunda mitad del siglo XX ¿hay un problema de espacio para el depósito de los documentos? ¿Se ha propuesto alguna solución?

3. ¿Qué ventajas incorporan las TIC en la gestión documental?

4. ¿Conoces alguna normativa jurídica o de buenas prácticas para la gestión de los documentos?

El debate en el aula sirvió para que los alumnos asimilaran el cambio de paradigma en la gestión de los documentos, y la incidencia que han tenido las TIC en la actual administración y en los servicios al ciudadano.

Tras el visionado del audiovisual y el debate en el aula se les formularon las siguientes cuestiones, unas de respuesta cerrada, y otras de respuesta abierta.

1. ¿El audiovisual ayuda a identificar características de la gestión documental?

2. El debate en clase, después de visualizar el audiovisual, ¿ayuda a clarificar conceptos de la gestión documental?

3. ¿Conoces alguna película relacionada con los documentos de archivo?

4. ¿Crees que las redes sociales pueden difundir la labor e importancia de los archivos de las organizaciones? 
Posteriormente se continuó debatiendo en clase, y los alumnos tuvieron oportunidad de aportar su visión personal sobre las distintas cuestiones formuladas.

\section{Resultados obtenidos}

En la actividad docente participaron ocho alumnos (la totalidad de la clase en esta asignatura optativa). Consideramos que es una muestra poco representativa en relación al total de los alumnos del grado, y que no se puede generalizar a ninguna población los resultados. Sin embargo, sí que nos aporta información sobre la utilización de audiovisuales en el aula, y entendemos que en el futuro con una muestra mayor podremos obtener resultados más representativos. Después de una hora de debate en el aula se les pasó un cuestionario para ver el interés de la actividad, en una escala de cuatro valores (mucho, regular, poco o nada), así como una respuesta abierta relacionada con la temática.

Como se observa en la Figura 2, la mitad de los alumnos consideran que el audiovisual ayuda mucho a identificar características de la gestión documental, y la otra mitad, que ayuda de forma regular. En ningún caso contestan que poco o nada, con lo que se considera que sí que les resulta de ayuda el visionado de un audiovisual relacionado con la cuestión que se trata.

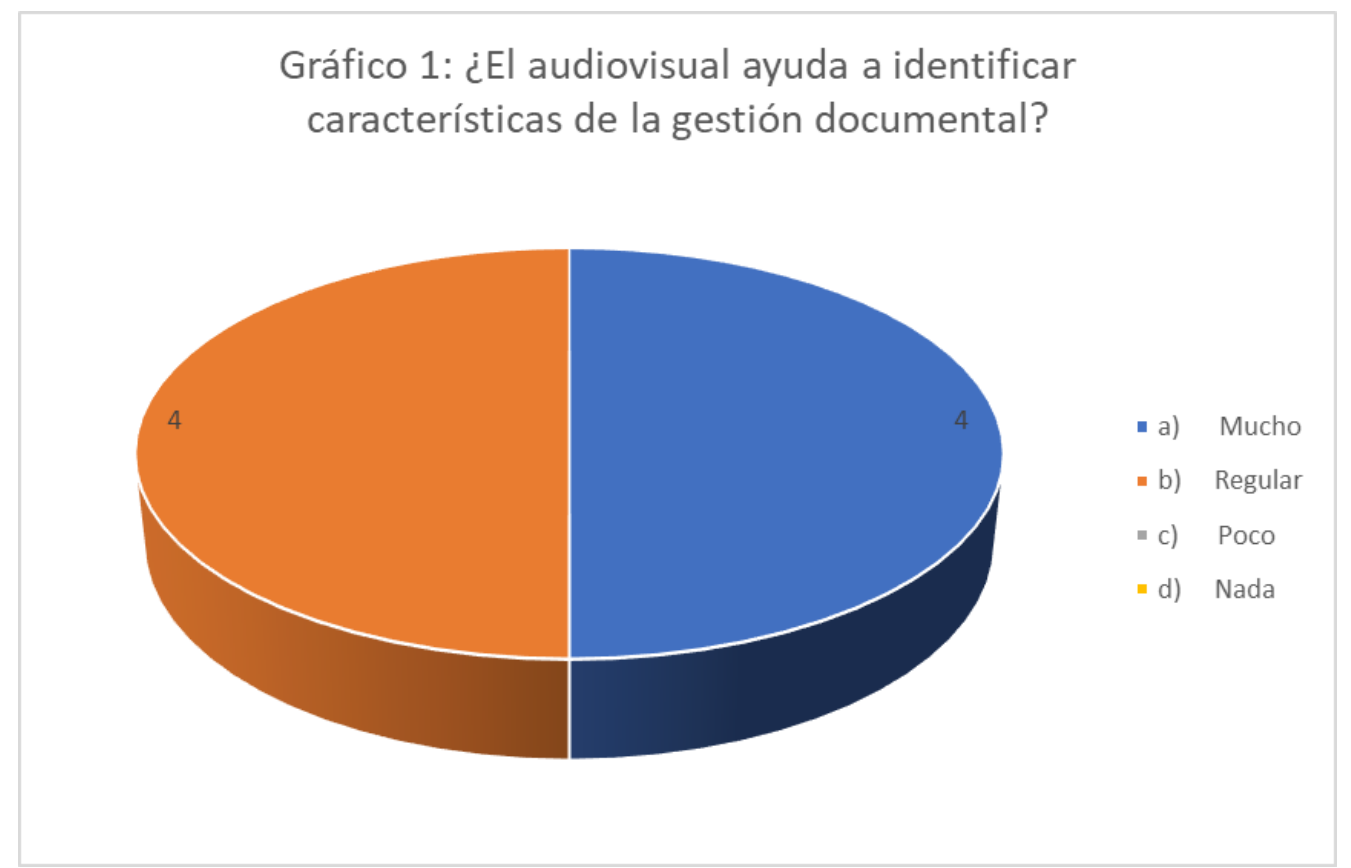

Figura. 2. Respuestas obtenidas sobre la pregunta $¿ E l$ audiovisual ayuda a identificar características de la gestión documental? Fuente: elaboración propia. 
El alumnado concede más importancia al debate posterior al visionado del audiovisual, ya que la totalidad de ellos contestaron que mucho, como se observa en la Figura 3.

\section{Gráfico 2: El debate en clase, después de visualizar el audiovisual, ¿̇ayuda a clarificar conceptos de la gestión documental?}

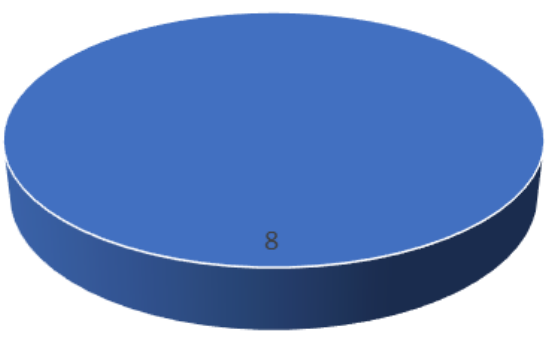

- a) Mucho

- b) Regular

- c) Poco

- d) Nada

Figura. 3. Unanimidad del alumnado sobre la consulta respecto al debate en clase posterior al visionado del audiovisual. Fuente: elaboración propia.

Respecto a la relevancia de las redes sociales para dar a conocer la labor de las organizaciones, que también se observa en el documental, prácticamente la totalidad del alumnado considera que mucho, excepto una persona que contestó que regular. De ello se desprende que las redes sociales resultan positivas y adecuadas para dar a conocer la gestión documental que se realiza en los archivos. El porcentaje se puede contemplar en la Figura 4.

Gráfico 3: ¿Crees que las redes sociales pueden difundir la labor e importancia de los archivos de las organizaciones?

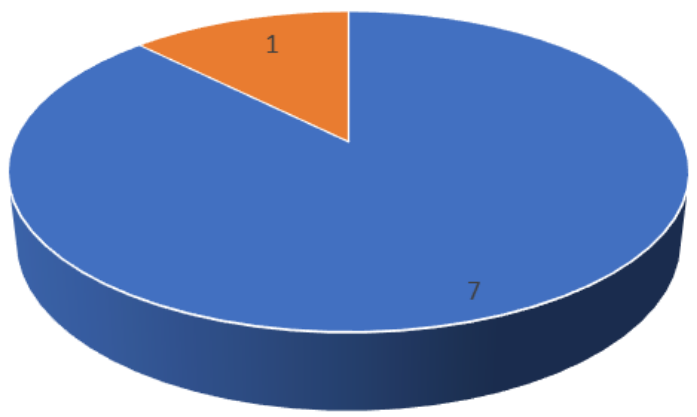

- a) Mucho

- b) Regular

- c) Poco

- d) Nada

Figura. 4. Porcentaje altamente significativo sobre la importancia de las redes sociales para la difusión de la actividad de los archivos. Fuente: elaboración propia. 


\section{Conclusiones}

Para terminar de valorar esta actividad los alumnos tenían la opción voluntaria de responder a una pregunta abierta para opinar sobre películas que trataran sobre archivos. Tan sólo contestaron dos con el aporte de dos películas muy conocidas, a la que contestaron sólo dos alumnos, indicando que sólo conocían las películas "Ángeles y Demonios" y "Los archivos del pentágono".

La totalidad de alumnado considera que el debate posterior a la visualización del audiovisual es altamente positivo, ya que permite que se puedan aportar distintos puntos de vista relacionados con la temática de la gestión del documento.

La experiencia desarrollada, aunque se ha realizado con un número bajo de alumnado y puede ser poco representativa, resulta altamente satisfactoria porque nos ha permitido conocer la percepción de la utilización de un audiovisual con notas de ciencia ficción en la gestión del documento electrónico.

\section{Referencias}

Abreus González, A., \& Haro Calero, R. D. (2019). El empleo de materiales auténticos audiovisuales para el desarrollo la expresión oral en inglés: estudio de caso en Ecuador. EDMETIC, 8(1), 23-35. https://doi.org/10.21071/edmetic.v8i1.10695

Cabedo Mallol, V., Casar Furió, M. E., Giménez Chornet, V., Oltra Gutiérrez, J. V. y Ramón Fernández, F. (2016). Casos prácticos jurídicos basados en series de animación. Francisca Ramón Fernández (coord.). Valencia: Tirant lo Blanch.

Cabedo Mallol, V., Casar Furió, M. E., Giménez Chornet, V., Lull Noguera, C., Oltra Gutiérrez, J. V. y Ramón Fernández, F. (2017). Casos prácticos jurídicos basados en series de ficción. Francisca Ramón Fernández (coord.), Valencia, Tirant lo Blanch.

Cabedo Mallol, V., Casar Furió, M. E., Giménez Chornet, V., Lull Noguera, C., Oltra Gutiérrez, J. V. y Ramón Fernández, F. (2018). Casos prácticos jurídicos basados en documentales. Francisca Ramón Fernández (coord.). Valencia: Tirant lo Blanch.

Cabedo Mallol, V., Casar Furió, M. E., Giménez Chornet, V., Lull Noguera, C., Oltra Gutiérrez, J. V., Osete Cortina, L., Ramón Fernández, F. y Soriano Soto, Mạ. D. (2019). Casos prácticos jurídicos basados en el humor. Francisca Ramón Fernández (coord.). Valencia: Tirant lo Blanch.

Giménez Chornet, V. (2014). La Innovación en los archivos de España y la Unión Europea. Métodos de información, 5(8), 75-95, http://dx.doi.org/10.5557/IIMEI5N8-075095

Hollist, A. (2012). Council for Administration: higher apprenticeships. Higher Education, Skills and Work-Based Learning, 2(3), 309-316. https://doi.org/10.1108/20423891211271700 
Montero Caro, M. D. (2020). El derecho de acceso a la información pública desde el prisma constitucional. Métodos de Información, 11(21), 42-58. https://dx.doi.org/10.5557/IIMEl11-N21-042058

Oltra Gutiérrez, J. V., Ramón Fernández, F., Cabedo Mallol, V., Casar Furió, M. E., y Giménez Chornet, V. (2016). Uso de fragmentos de películas y series como herramientas de innovación docente. Una experiencia con alumnos de informática. INRED. II Congreso Nacional de Innovación Educativa y Docencia en Red (1-11), Valencia, Universitat Politècnica de València, https://n9.cl/1abo4

Ramón Fernández, F., Cabedo Mallol, V., Casar Furió, M. E., Giménez Chornet, V., Lull Noguera, C. y Oltra Gutiérrez, J. V. (2016a). Diseño de casos prácticos jurídicos basados en series de animación. Una innovación docente con soporte audiovisual. Derecho y TIC: últimas innovaciones docentes, Coordinadores Ana María Delgado García e Ignacio Beltrán de Heredia Ruiz (123-132). Barcelona: Huygens editorial.

Ramón Fernández, F., Cabedo Mallol, V., Casar Furió, M. E., Giménez Chornet, V., y Oltra Gutiérrez, J. V. (2016b). Utilización de las series de animación como recurso didáctico en la elaboración de casos. Análisis de una experiencia en la innovación docente en la Universitat Politècnica de València. INRED. II Congreso Nacional de Innovación Educativa y Docencia en Red (1-12), Universitat Politècnica de València. https://n9.cl/m5em

Ramón Fernández, F., Cabedo Mallol, V., Casar Furió, M. E., Giménez Chornet, V., Lull Noguera, C. y Oltra Gutiérrez, J. V. (2018a). "La utilización de los documentales como herramienta para la evaluación de la competencia transversal CT07. Responsabilidad ética, medioambiental y profesional. Experiencias en la docencia impartida en la Universitat Politècnica de València. Derecho y TIC: últimas innovaciones docentes, Coordinadores Ana María Delgado García e Ignacio Beltrán de Heredia Ruiz (193-204), Barcelona: Huygens editorial.

Ramón Fernández, F., Cabedo Mallol, V., Casar Furió, M. E., Giménez Chornet, V., Lull Noguera, C. y Oltra Gutiérrez, J. V. (2018b). La utilización de los documentales como herramienta para la evaluación de la competencia transversal CT07. Responsabilidad ética, medioambiental y profesional. Jornada de Innovación Docente ETSINF 2018 (1-2), Valencia: Universitat Politècnica de València.

Ramón Fernández, F., Cabedo Mallol, V., Casar Furió, M. E., Giménez Chornet, V., Lull Noguera, C., Oltra Gutiérrez, J. V. y Orduña Malea, E. (2018c). El audiovisual Tutankamón como herramienta para la evaluación de la competencia transversal de pensamiento crítico y responsabilidad ética medioambiental y profesional. Una experiencia en la Universitat Politècnica de València. IN-RED 2018 (1-12), Valencia. Universitat Politècnica de València. https://n9.cl/cehlw

Ramón Fernández, F., Lull Noguera, C., Soriano Soto, Mª̣. D., Cabedo Mallol, V., Casar Furió, Ma‥ E., Giménez Chornet, V., Oltra Gutiérrez, J. V. y Orduña Malea, E. (2019a). "Diseño de materiales docentes basados en recursos audiovisuales de humor para la mejora del aprendizaje y su evaluación en ciencias sociales y jurídicas. Análisis del diseño de la actividad sobre las casas Cueva y «Los Picapiedra». La docencia del Derecho en la sociedad digital. Barcelona: Huygens Editorial. 


\section{Geduweb}

Revista de Tecnología de Información y Comunicación en Educación • Volumen 15, Nㅜ 2. Mayo-agosto 2021

Ramón Fernández, F., Lull Noguera, C., Soriano Soto, Ma․ D. (2019b). Actividades docentes del PYME. Diseño de materiales docentes para la mejora del aprendizaje y su evaluación en ciencias del suelo, sociales y jurídicas. XXXII Reunión Nacional de Suelos, 10 a 13 de septiembre de 2019 RENS, Sevilla. Sociedad Española de la Ciencia del Suelo.

Sebastià Salat, M. (2009). La transformación de los archivos y de la Archivística: el nuevo paradigma desde la hibridez a la metadisciplina y la metacomunidad profesional. Tabula. Revista de archivos de Castilla y León, №. 12, 17-32. 